Supporting Information

\title{
Controllable Synthesis of CuS Nanostructures from Self-assembled Precursors with Assist of Biomolecule
}

\author{
Benxia Li, Yi Xie, * Yi Xue \\ Department of Nanomaterials and Nanochemistry, Hefei National Laboratory for \\ Physical Sciences at Microscale, University of Science and Technology of China, Hefei, \\ Anhui 230026, P. R. China
}

*To whom correspondence should be addressed. 


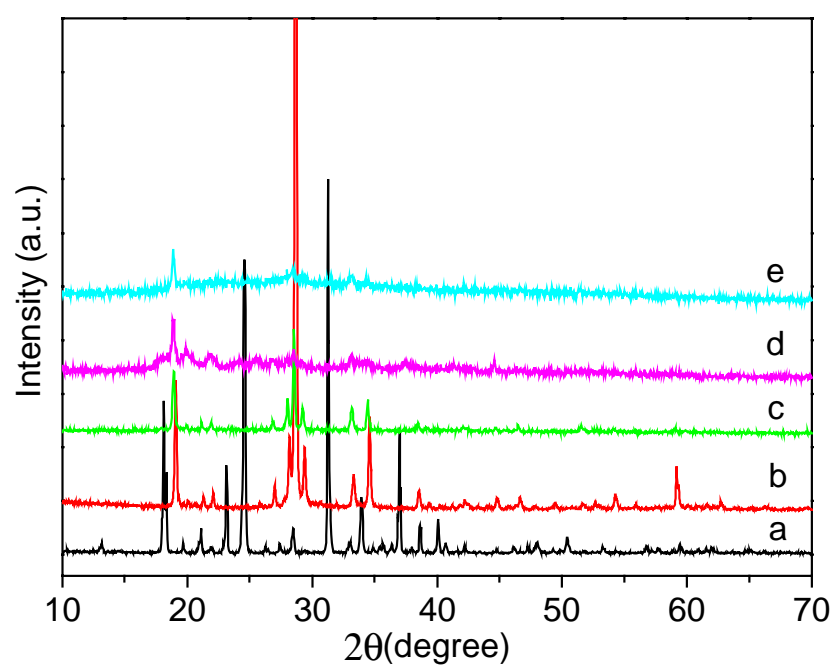

Figure S1. XRD patterns of (a) pure L-cysteine used as a reagent in the present experiment; (b) pure L-cystine purchased from the Shanghai Chemical Company; (c-e) the as-formed precursors (c: precursor A, d: precursor B, e: precursor C) centrifuged from the reaction solutions and dried.

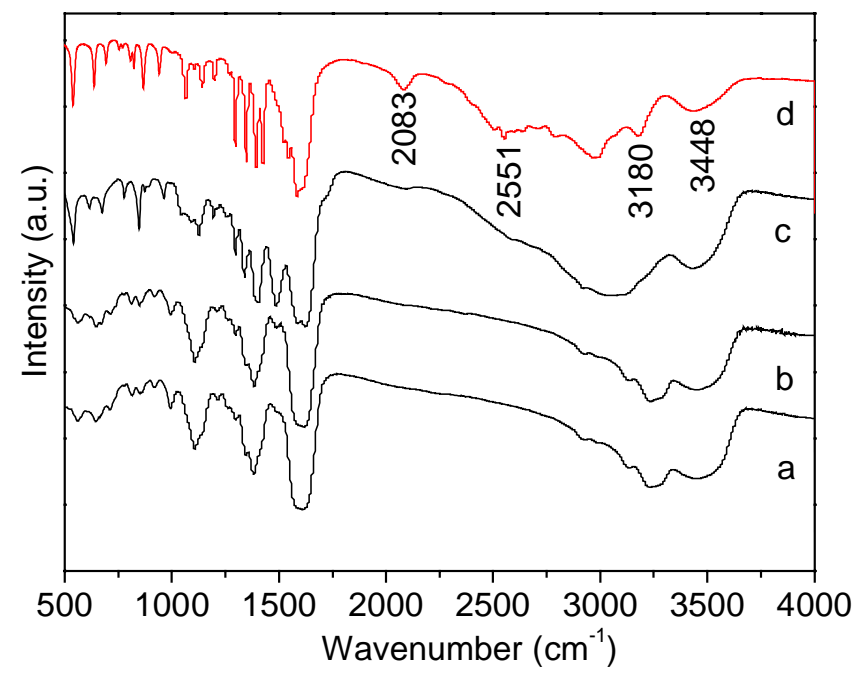

Figure S2. Fourier transform infrared (FTIR) spectra of the as-formed precursors and pure L-cysteine used in the present experiment: (a) precursor A, (b) precursor B, (c) 
precursor C, and (d) pure L-cysteine.

XRD patterns of the three precursors present following evidential information. Firstly, XRD patterns of the precursors are different from that of pure L-cysteine, indicating that new structures have formed to assemble into the precursors. Secondly, the observable corresponding diffraction peaks in the XRD patterns of the three precursors are located at the same positions with that in the XRD pattern of the pure L-cystine, indicating that the three precursors consist of the same crystalline component of L-cystine. Thirdly, the weaker and broader diffraction peaks in the XRD patterns of precursor B and precursor $\mathrm{C}$ indicate that they are weakly crystalline or may contain lots of amorphous composition. It is presumed that the precursors are likely composed of some small crystallites of L-cystine molecules and amorphous $\mathrm{Cu}(\mathrm{I})$-cysteine complexes. The weakly crystalline character of precursor B and precursor C should be caused by larger numbers of amorphous $\mathrm{Cu}(\mathrm{I})$-cysteine complexes in them. In the Fourier transform infrared (FTIR) spectra of L-cysteine and the as-obtained precursors, the bands at $3448 \mathrm{~cm}^{-1}$ and $3180 \mathrm{~cm}^{-1}$ can be assigned to the $-\mathrm{OH}$ and $-\mathrm{NH}_{2}$ stretching vibrations. It was found that the characteristic signal of $-\mathrm{SH}$ at about $2551 \mathrm{~cm}^{-1}$ disappeared in the spectra of the precursors, suggesting that the - $\mathrm{SH}$ group of L-cysteine has changed in the precursors. ${ }^{1}$ From $1800 \mathrm{~cm}^{-1}$ to $900 \mathrm{~cm}^{-1}$ in pure L-cysteine, the bands assigned to the stretching vibrations of C-S bonds are weakened in the precursors due to the formation of $\mathrm{Cu}-\mathrm{S}$ bond. ${ }^{1,2}$ The preliminary proof provided by XRD patterns and FTIR spectra further support the conclusion that the precursors are likely assembled by the small crystallites of L-cystine molecules and the 
amorphous $\mathrm{Cu}(\mathrm{I})$-cysteine complexes.

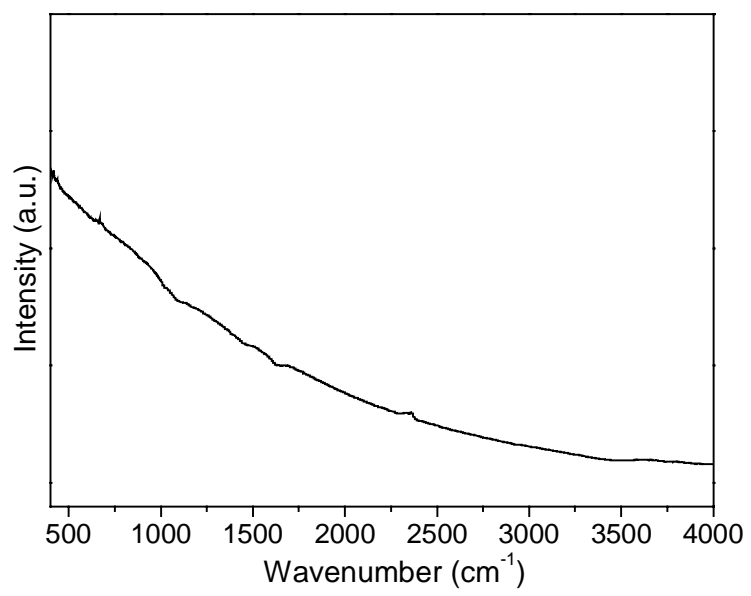

Figure S3. FTIR spectrum of the final $\mathrm{CuS}$ product. There is not any detectable band in the FTIR spectrum, indicating that the precursors have completely decomposed and $\mathrm{CuS}$ is obtained by removing the dissoluble carbonous byproducts.

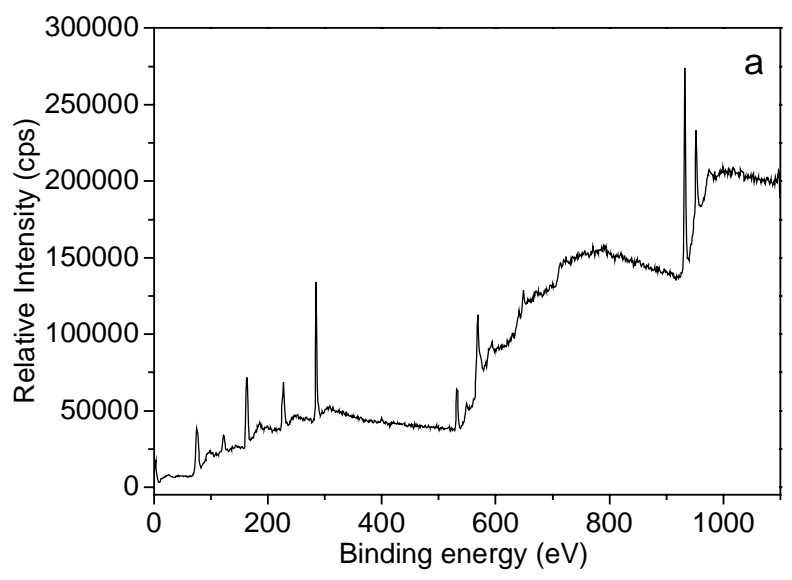



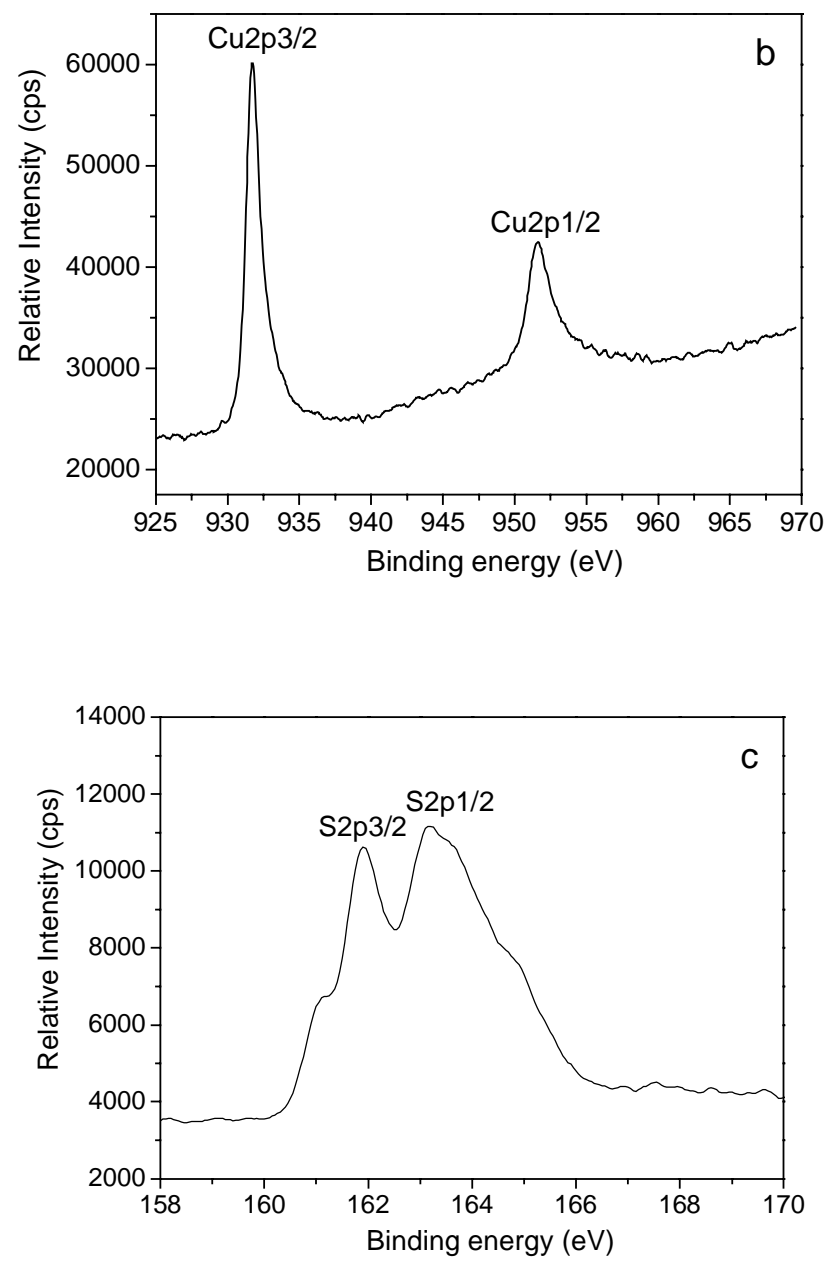

Figure S4. X-Ray photoelectron spectroscopy (XPS) spectra of (a) survey, (b) $\mathrm{Cu} 2 \mathrm{p}$ and (c) S 2p in the as-obtained CuS samples.

The XPS spectra further confirmed the composition of the compound. ${ }^{3}$ The survey spectrum indicates the presence of $\mathrm{Cu}, \mathrm{S}, \mathrm{C}$, and $\mathrm{O}$ peaks where the $\mathrm{C}$ is from the reference and $\mathrm{O}$ is due to the absorption of air on the surface of the porous CuS hollow microspheres. The high-resolution spectrum in the $\mathrm{Cu} 2 \mathrm{p}$ region revealed the presence of two strong peaks at 931.7 and $951.6 \mathrm{eV}$ for $\mathrm{Cu} 2 \mathrm{p} 3 / 2$ and $\mathrm{Cu} 2 \mathrm{p} 1 / 2$, respectively. Another high-resolution spectrum in the $\mathrm{S} 2 \mathrm{p}$ region showed the presence of a doublet peak at $162 \mathrm{eV}$. All these results are well matched with the reported values. ${ }^{4}$ 


\section{References}

1. (a) Pretsch, E.; Clerc, T.; Seibl, J.; Simon, W. In Tables of Spectral Data for Structure Determination of Organic Compounds, 2nd ed.; Translated by Biemann, K.; Springer-Verlag, New York, 1989; pp I5. (b) Panigrahi, S.; Kundu, S.; Basu, S.; Praharaj, S.; Jana, S.; Pande, S.; Nanotechnology 2006, 17, 5461.

2. Yang, J.; Zeng, J. H.; Yu, S. H.; Yang, L.; Zhang, Y. H.; Qian, Y. T.; Chem. Mater. 2000, 12, 2924.

3. (a) Deroubaix, G.; Marcus, P.; Surf. Interface Anal., 1992, 18, 39. (b) Practical Surface Analysis by Auger and X-ray Photoelectron Spectroscopy, ed. Briggs, D. and Searl, M. P.; John Wiley \& Sons Ltd., New Jersey, 1983.

4. Wu, C. Y.; Yu, S. H.; Chen, S. F.; Liu, G. N.; Liu, B. H.; J. Mater. Chem. 2006, 16, 3326. 